ARTICLE

DOI: $10.1038 / s 41467-018-05947-2$

\title{
Near-infrared-triggered photon upconversion tuning in all-inorganic cesium lead halide perovskite quantum dots
}

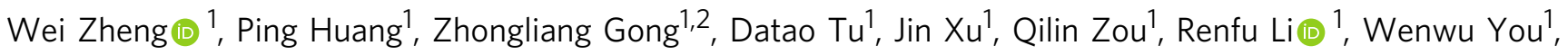
Jean-Claude G. Bünzli id ${ }^{3}$ \& Xueyuan Chen (1) ${ }^{1,2}$

All-inorganic $\mathrm{CsPbX}_{3}(\mathrm{X}=\mathrm{Cl}, \mathrm{Br}$, and I) perovskite quantum dots ( $\mathrm{PeQDs}$ ) have shown great promise in optoelectronics and photovoltaics owing to their outstanding linear optical properties; however, nonlinear upconversion is limited by the small cross-section of multiphoton absorption, necessitating high power density excitation. Herein, we report a convenient and versatile strategy to fine tuning the upconversion luminescence in $\mathrm{CsPbX}_{3}$ PeQDs through sensitization by lanthanide-doped nanoparticles. Full-color emission with wavelengths beyond the availability of lanthanides is achieved through tailoring of the PeQDs bandgap, in parallel with the inherent high conversion efficiency of energy transfer upconversion under low power density excitation. Importantly, the luminescent lifetimes of the excitons can be enormously lengthened from the intrinsic nanosecond scale to milliseconds depending on the lifetimes of lanthanide ions. These findings provide a general approach to stimulate photon upconversion in PeQDs, thereby opening up a new avenue for exploring novel and versatile applications of PeQDs.

\footnotetext{
${ }^{1}$ CAS Key Laboratory of Design and Assembly of Functional Nanostructures, and Fujian Key Laboratory of Nanomaterials, Fujian Institute of Research on the Structure of Matter, Chinese Academy of Sciences, Fuzhou 350002 Fujian, China. ${ }^{2}$ University of Chinese Academy of Sciences, 100049 Beijing, China. ${ }^{3}$ Institute of Chemical Sciences and Engineering, Swiss Federal Institute of Technology, Lausanne (EPFL), CH-1015 Lausanne, Switzerland. Correspondence and requests for materials should be addressed to X.C. (email: xchen@fjirsm.ac.cn)
} 
A ll-inorganic cesium lead halide $\left(\mathrm{CsPbX}_{3}, \mathrm{X}=\mathrm{Cl}, \mathrm{Br}\right.$, and I) perovskite quantum dots (PeQDs) have recently attracted intensive attention in a wide array of research fields, owing to their outstanding physicochemical properties such as large absorption coefficients, high photoluminescence (PL) quantum yields (QYs), narrow emission bands, and tunable bandgap and PL emissions by varying the halide composition ${ }^{1-4}$. These elegant characteristics make $\mathrm{CsPb}_{3} \mathrm{PeQDs}$ ideal candidates as light-harvesting or light-emitting materials for diverse optoelectronic and photovoltaic applications $s^{5-12}$. Although PeQDs exhibit excellent linear optical properties under ultraviolet (UV) or visible light excitation, their nonlinear optical properties such as near-infrared (NIR)-triggered photon upconversion are limited by low efficiency $\left(<10^{-8}\right)$ of multiphoton absorption and the requirement of expensive pulsed lasers for excitation ${ }^{13-18}$. In contrast to linear absorption and emission, the nonlinear upconversion analogs feature a series of advantages including a large penetration depth, high spatial resolution, minimal background interference, and little damage to the targeted samples, which hold great promise in areas as diverse as multiplexed optical encoding, three-dimensional displays, super-resolution bioimaging, and effective solar spectrum conversion ${ }^{19-26}$.

As compared with PeQDs, lanthanide-doped nanoparticles (NPs) are much more efficient $\left(10^{-1}-10^{-3}\right)$ in photon upconversion through successive photon absorption and energy transfer processes within lanthanide ions, and thus can be excited by using a low-cost continuous-wave (CW) diode lase ${ }^{27-30}$. By controlling the energy transfer processes based on core/shell engineering, these NPs can produce efficient upconversion luminescence (UCL) with wavelengths spanning from UV to NIR and lifetimes ranging from microseconds to milliseconds ${ }^{31-36}$. Nevertheless, upconversion tuning in lanthanide-doped NPs requires cumbersome experimentation involving repeated trials to optimize the dopant concentration ${ }^{37}$. Moreover, there are inherent limitations on wavelength tunability in lanthanide-doped NPs due to the defined and discrete energy levels of lanthanide ions.

To circumvent these limitations, it is essential to bring together both advantages of lanthanide-doped NPs and PeQDs, whereby lanthanide-doped NPs may serve as an effective sensitizer for PeQDs to boost their upconversion efficiency, whereas PeQDs with continuously tunable emission bands could extend the emission wavelengths of lanthanide-doped NPs. Unfortunately, previous endeavors on this aspect through non-radiative Förster resonance energy transfer (FRET) from lanthanide-doped NPs to quantum dots usually resulted in serious quenching of overall UCL intensity and negligibly weak quantum dots emissions, because it is notoriously difficult to control the number of energy acceptors in close proximity of the donors within an effective FRET distance ${ }^{38-40}$.

Herein, we report a convenient and versatile approach to fine tuning the UCL in $\mathrm{CsPb}_{3} \mathrm{PeQDs}$ through sensitization by lanthanide-doped NPs. We demonstrate that the sensitization is governed by a radiative energy transfer upconversion (RETU) process. In our design (Fig. 1), lanthanide-doped NPs function as the energy donor to convert the NIR excitation light into the UV and visible emission light through successive photon absorption and energy transfer upconversion processes within lanthanides. The emission light from the NPs is then reabsorbed by PeQDs to create electron-hole pairs (excitons) in the conduction band and valence band, followed by photon emission through exciton recombination. A key feature of RETU is that the PeQDs possess large absorption coefficient and high PLQY. As a result, tunable upconversion emission with wavelengths beyond the availability of lanthanide-doped NPs is likely to be realized via bandgap tailoring of PeQDs, in parallel with the benefits of lifetime tunability by lanthanide ions and the inherent high conversion efficiency of energy transfer upconversion under low power density excitation.

\section{Results}

Synthesis and characterization. High-quality $\mathrm{CsPbX}_{3} \mathrm{PeQDs}$ were synthesized through a facile and novel method by injecting $\mathrm{HX}(\mathrm{X}=\mathrm{Cl}, \mathrm{Br}$, and I) to the precursor solution of Cs-oleate and $\mathrm{Pb}$ (II)-oleate at an elevated temperature (Methods). Structure and morphology characterizations through transmission electron microscopy (TEM), X-ray powder diffraction, energy dispersive $\mathrm{X}$-ray spectra, and selected area electron diffraction revealed cubic phase and high crystallinity of the PeQDs with particle sizes of $11.0-13.3 \mathrm{~nm}$ (Fig. 2a, b and Supplementary Figs. 1 and 2). Optical absorption spectra showed that the PeQDs had large absorbance in the UV and visible spectral region with band edges shifting from $410 \mathrm{~nm}$ to $700 \mathrm{~nm}$ as the halide composition changed from $\mathrm{Cl}^{-}$to $\mathrm{I}^{-}$(Fig. 2c), indicating bandgap tailoring of PeQDs by adjusting the halide composition ${ }^{41}$. Upon UV excitation at $365 \mathrm{~nm}$, these PeQDs displayed remarkably bright PL with full-gamut color tuning from violet to green and deep red (Fig. 2d). PL spectra of the PeQDs exhibited tunable emissionband, which can be ascribed to the band-edge exciton

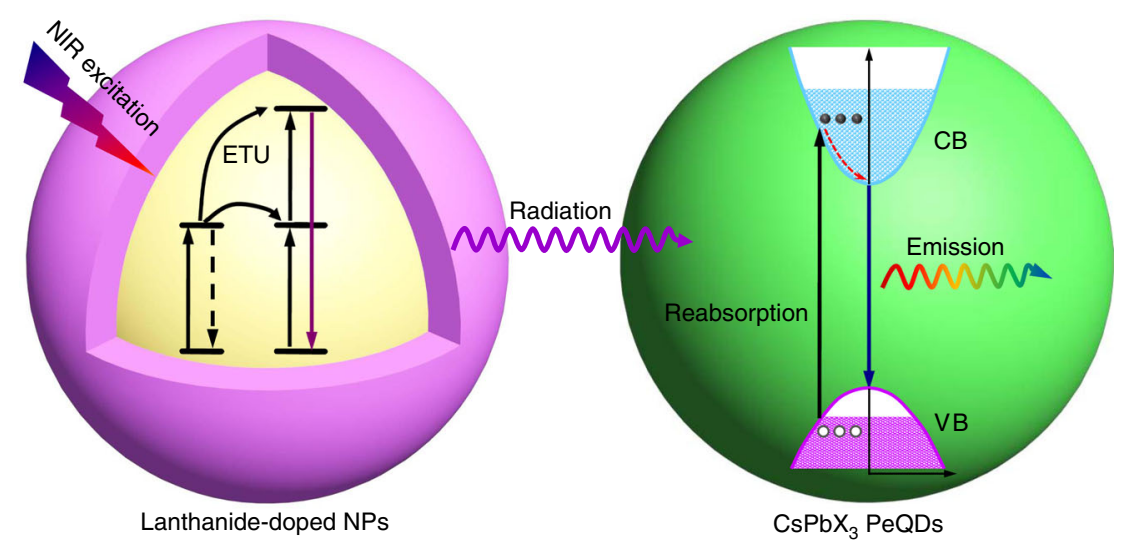

Fig. 1 Schematic representation of the radiative energy transfer upconversion (RETU) processes in all-inorganic CsPbX ${ }_{3}$ perovskite quantum dots (PeQDs) through sensitization by lanthanide-doped nanoparticles (NPs). Lanthanide-doped NPs function as the energy donor to convert the NIR excitation light into the ultraviolet (UV) and visible emission light through successive photon absorption and energy transfer upconversion (ETU) processes within lanthanides. The emission light from the NPs is then reabsorbed by PeQDs to create electron-hole pairs (excitons) in the conduction band (CB) and valence band (VB), followed by photon emission through exciton recombination. The solid and dash lines represent the electronic transitions 

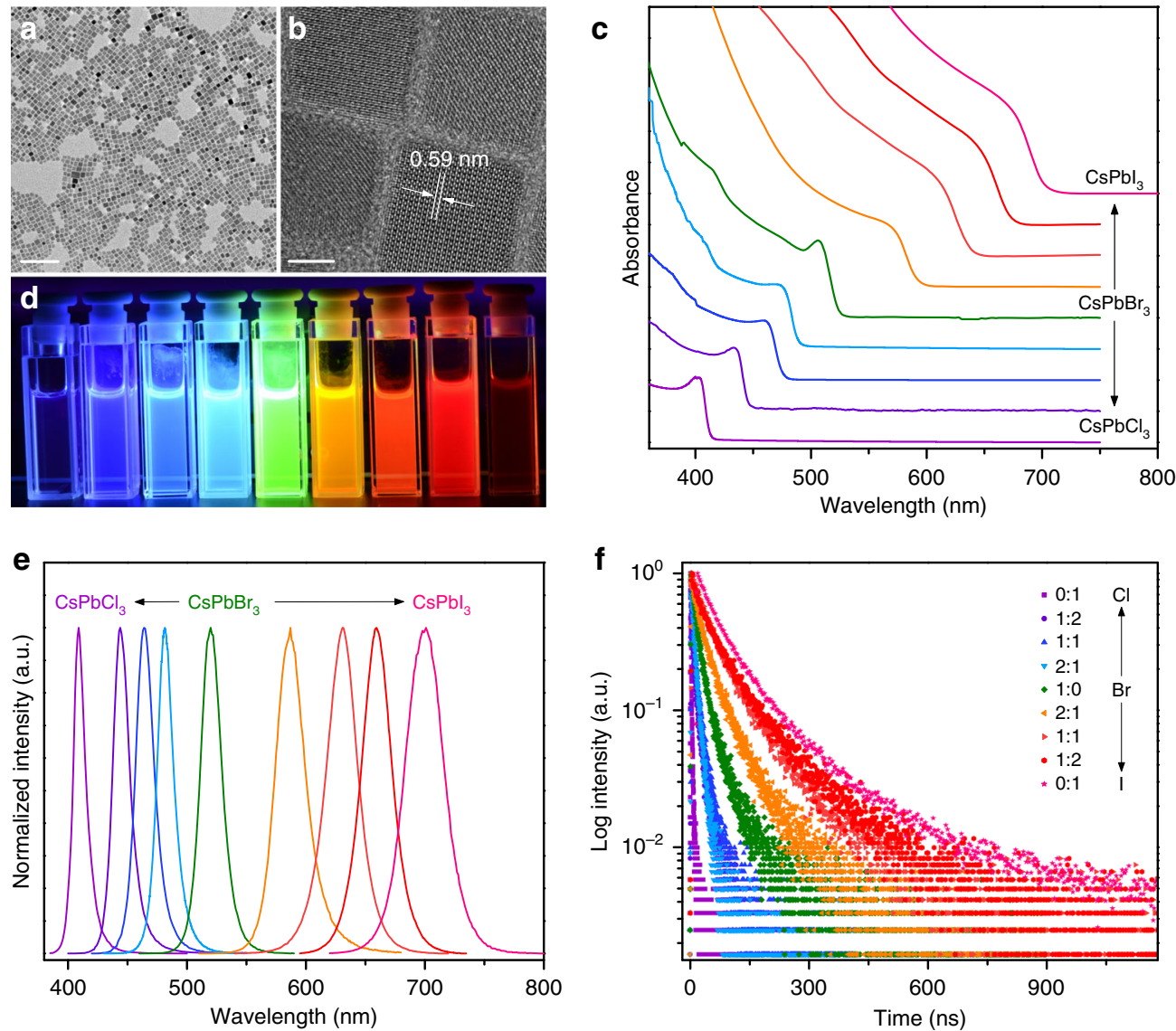

Fig. 2 Synthesis and characterization of $\mathrm{CsPbX}_{3}$ perovskite quantum dots ( $\mathrm{PeQDs}$ ). a Transmission electron microscopy (TEM) and $\mathbf{b}$ high-resolution TEM images of the as-synthesized $\mathrm{CsPbBr}_{3}$ PeQDs. The scale bars in (a) and (b) represent $100 \mathrm{~nm}$ and $5 \mathrm{~nm}$, respectively. c Optical absorption spectra, d photoluminescence (PL) photograph $(\lambda=365 \mathrm{~nm})$, e PL spectra $(\lambda=360 \mathrm{~nm})$ and $\mathbf{f}$ PL decays of the as-synthesized CsPbX ${ }_{3}$ PeQDs with varying halide compositions

recombination over the entire visible spectral region ${ }^{42}$, with bandwidths of 11.7-37.0 nm (Fig. 2e and Supplementary Table 1). PL decays indicated effective PL lifetimes of the band-edge excitons in the range of 1.8-81.1 ns with faster decay for widerbandgap PeQDs (Fig. 2f and Supplementary Table 2). The absolute PLQYs, defined as the ratio of the number of emitted photons to the number of absorbed photons, were determined to be $26.0-79.8 \%$ with the highest value of $79.8 \%$ for $\mathrm{CsPbBr}_{3}$ PeQDs (Supplementary Table 1).

We synthesized $\mathrm{LiYbF}_{4}: \mathrm{Tm}^{3+} @ \mathrm{LiYF}_{4}$ core/shell NPs through a high-temperature co-precipitation method (Supplementary Note 1) and selected them as the energy donors in view of their intense upconversion emission at $362 \mathrm{~nm}$ that can potentially excite the whole series of $\mathrm{CsPbX}_{3} \mathrm{PeQDs}^{43}$. The as-synthesized NPs were rhombohedral and highly crystallized with a mean size of $(26.7 \pm 1.2) \times(33.3 \pm 1.8) \mathrm{nm}$ and a shell thickness of $5.5 \pm 0.6$ $\mathrm{nm}$ (Supplementary Fig. 3). Under $980 \mathrm{~nm}$ excitation, the NPs displayed intense UCL with a set of sharp and typical emission peaks from $\mathrm{Tm}^{3+}$, which were divided into four regions: the UV region with peaks at $347 \mathrm{~nm}\left({ }^{1} \mathrm{I}_{6} \rightarrow{ }^{3} \mathrm{~F}_{4}\right)$ and $362 \mathrm{~nm}\left({ }^{1} \mathrm{D}_{2} \rightarrow\right.$ $\left.{ }^{3} \mathrm{H}_{6}\right)$, the blue region with peaks at $450 \mathrm{~nm}\left({ }^{1} \mathrm{D}_{2} \rightarrow{ }^{3} \mathrm{~F}_{4}\right)$ and 483 $\mathrm{nm}\left({ }^{1} \mathrm{G}_{4} \rightarrow{ }^{3} \mathrm{H}_{6}\right)$, the red region with single peak at $648 \mathrm{~nm}$ $\left({ }^{1} \mathrm{G}_{4} \rightarrow{ }^{3} \mathrm{~F}_{4}\right)$, and the NIR region with a peak at $792 \mathrm{~nm}\left({ }^{3} \mathrm{H}_{4} \rightarrow\right.$ ${ }^{3} \mathrm{H}_{6}$ ) (see Fig. 3a for a simplified diagram of transitions in the $\mathrm{Yb}^{3+}-\mathrm{Tm}^{3+}$ system and Supplementary Fig. 4) ${ }^{43}$. Power dependence investigations revealed that at least four pump photons are needed to populate the ${ }^{1} \mathrm{D}_{2}$ level of $\mathrm{Tm}^{3+}$ to yield the UV emissions and three pump photons are required to feed the ${ }^{1} \mathrm{G}_{4}$ level to produce the blue and red emissions (Supplementary
Fig. 5) ${ }^{44}$. The UCL lifetimes were determined to be $217,253,447$, and $601 \mu$ s for the decays from ${ }^{1} \mathrm{I}_{6},{ }^{1} \mathrm{D}_{2},{ }^{1} \mathrm{G}_{4}$, and ${ }^{3} \mathrm{H}_{4}$ of $\mathrm{Tm}^{3+}$, respectively (Supplementary Fig. 6).

Full-color upconversion tuning in NP-sensitized $\mathrm{CsPbX}_{3}$ PeQDs. To sensitize photon upconversion in $\mathrm{CsPbX}_{3} \mathrm{PeQDs}$, we dispersed the PeQDs with $\mathrm{LiYbF}_{4}: 0.5 \% \mathrm{Tm}^{3+} @ \mathrm{LiYF}_{4}$ core/shell NPs in a homogeneous colloidal cyclohexane solution with a weight ratio of $2: 1$, in which the NPs can function as an internal UV or blue lamp to illuminate the PeQDs by utilizing the intense UCL of $\mathrm{Tm}^{3+}$ (Fig. 3a). Figure 3b shows the NP-sensitized UCL spectra for $\mathrm{CsPbX}_{3}$ PeQDs under $980 \mathrm{~nm} \mathrm{CW}$ diode laser excitation. Intriguingly, we observed that the characteristic band-edge exciton emissions from the PeQDs were dominant in the UCL spectra, whereas the emissions of $\mathrm{Tm}^{3+}$ from the NPs were selectively quenched in accordance with the PeQDs absorption, which indicates an efficient energy transfer from the NPs to PeQDs as CW laser cannot trigger photon upconversion in pure PeQDs (Supplementary Fig. 7) ${ }^{17}$. As a result, multicolor emission with color gamut beyond the three primary colors can be easily achieved by varying the halide composition in PeQDs (Fig. 3c). Moreover, because of the narrow bandwidths of the PeQDs emissions, highly saturated Red-Green-Blue colors can be generated, leading to a wide color gamut with a triangle in the Commission Internationale de l'Eclairage chromaticity diagram encompassing $140 \%$ of that defined in the National Television System Committee (NTSC) color standard (Fig. 3d and Supplementary Table 3$)^{45}$. Such a wide color gamut tuned by NPsensitized PeQDs is inaccessible by either lanthanide-doped NPs 
a

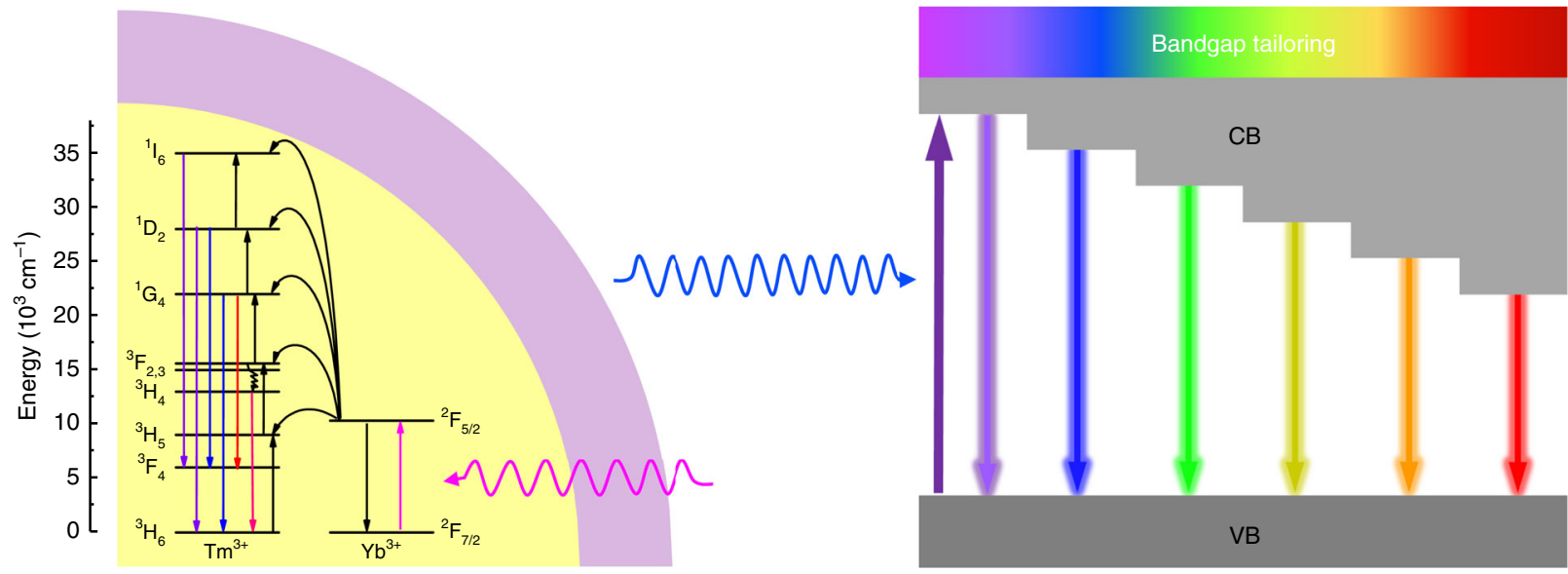

b

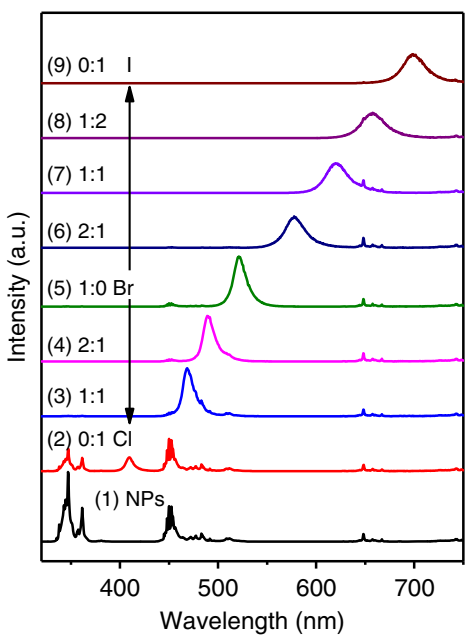

d

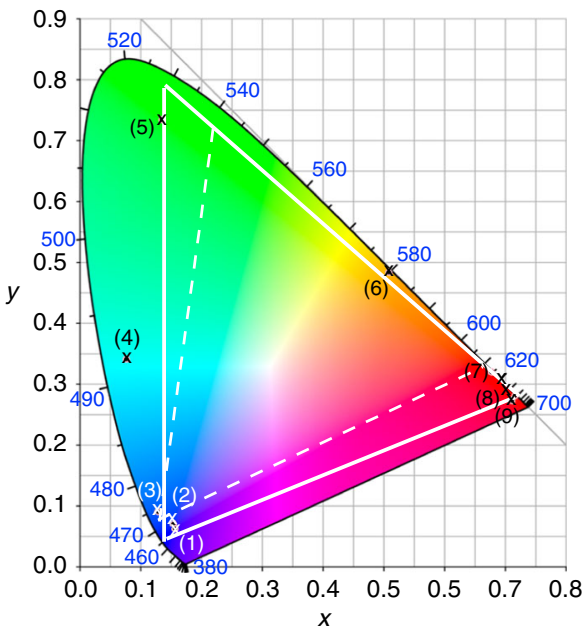

e

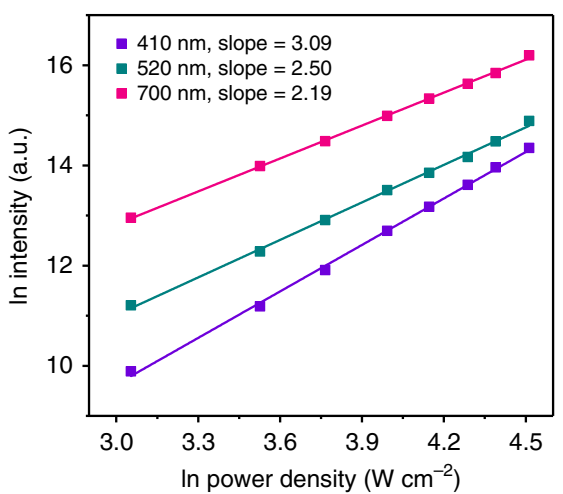

C
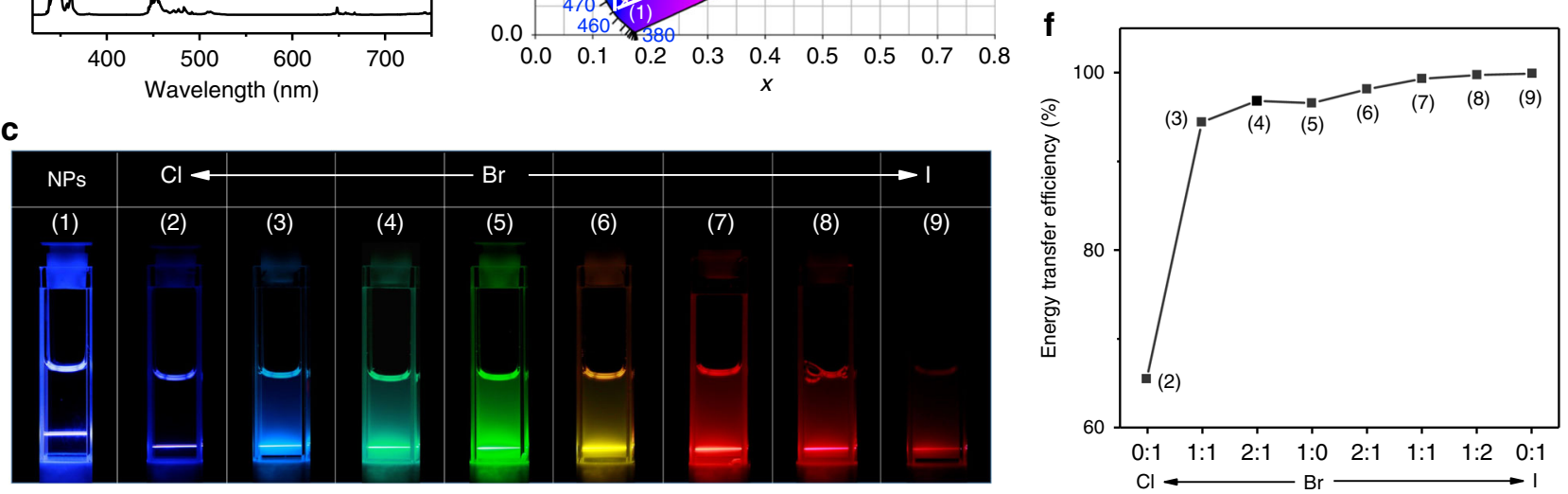

Fig. 3 Full-color upconversion tuning in $\mathrm{CsPbX}_{3}$ perovskite quantum dots ( $\mathrm{PeQDs}$ ) through sensitization by lanthanide-doped nanoparticles (NPs). a Simplified energy-level scheme of $\mathrm{LiYbF}_{4}: 0.5 \% \mathrm{Tm}^{3+} @ \mathrm{LiYF}_{4}$ core/shell NPs indicating major upconversion processes, and schematic illustration of full-color upconversion tuning in $\mathrm{CsPbX}_{3} \mathrm{PeQDs}$ through sensitization by the NPs. Black lines represent non-radiative energy transfer and the curved arrows denote internal conversion. b Upconversion luminescence (UCL) spectra for LiYbF $4: 0.5 \% \mathrm{Tm}^{3+} @$ LiYF 4 core/shell NPs and the NP-sensitized $\mathrm{CsPbX}_{3} \mathrm{PeQDs}\left(\mathrm{NPs}: 1 \mathrm{mg} \mathrm{mL}^{-1}\right.$; PeQDs: $2 \mathrm{mg} \mathrm{mL}^{-1}$ ) with varying halide compositions under $980 \mathrm{~nm}$ continuous-wave (CW) diode laser excitation at a power density of $50 \mathrm{~W} \mathrm{~cm}^{-2}$. c Photographs of samples (1-9) under $980 \mathrm{~nm}$ illumination, showing color tuning through bandgap tailoring of PeQDs. d Corresponding color gamut of the emission colors (solid white triangle) from the samples shown in (c), compared with the color gamut (dashed white triangle) defined in the NTSC television color standard. e Double logarithmic plots of intensity vs. excitation power for the upconverted exciton emissions from $\mathrm{CsPbCl}_{3}, \mathrm{CsPbBr}_{3}$, and $\mathrm{CsPbl}_{3} \mathrm{PeQDs}$ at 410, 520, and $700 \mathrm{~nm}$, respectively. $\mathbf{f}$ Calculated energy transfer efficiency in NP-sensitized PeQDs, as obtained from (b)

or PeQDs alone and is highly desired for optical coding and three-dimensional displays $46-48$.

Power dependence investigations reveal that the slope of the double logarithmic plots of intensity vs. excitation power is larger than 3 for the upconverted exciton emission from $\mathrm{CsPbCl}_{3}$ and larger than 2 for the emissions from $\mathrm{CsPbBr}_{3}, \mathrm{CsPb}_{3}$, and mixed $\mathrm{CsPb}(\mathrm{Cl} / \mathrm{Br})_{3}$ and $\mathrm{CsPb}(\mathrm{Br} / \mathrm{I})_{3}$ (Fig. 3e and Supplementary Fig. 8).
According to Pollnau et al. ${ }^{34}$, this may be interpreted as reflecting four-photon and three-photon processes, respectively, whereby energy transfer upconversion appears to be the dominant mechanism. It implies that, at least four pump photons are required to generate the UV emissions of $\mathrm{Tm}^{3+}$ to excite $\mathrm{CsPbCl}_{3}$, whereas only three pump photons are necessary to produce the blue or red emissions of $\mathrm{Tm}^{3+}$, which, in turn, 
a
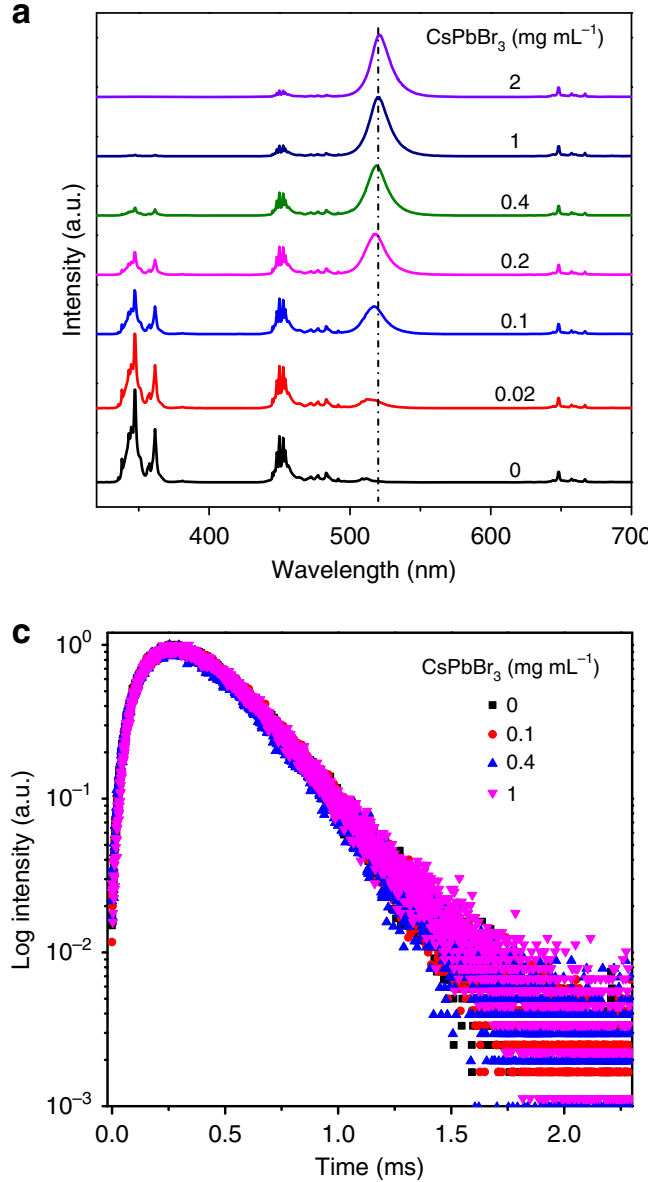

b

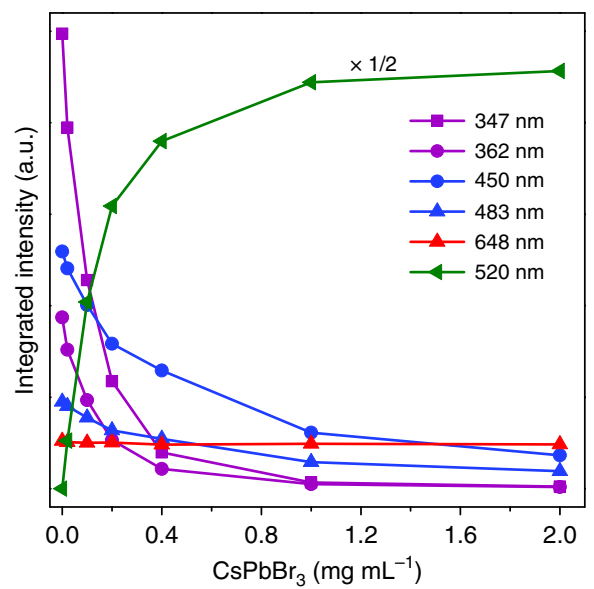

d

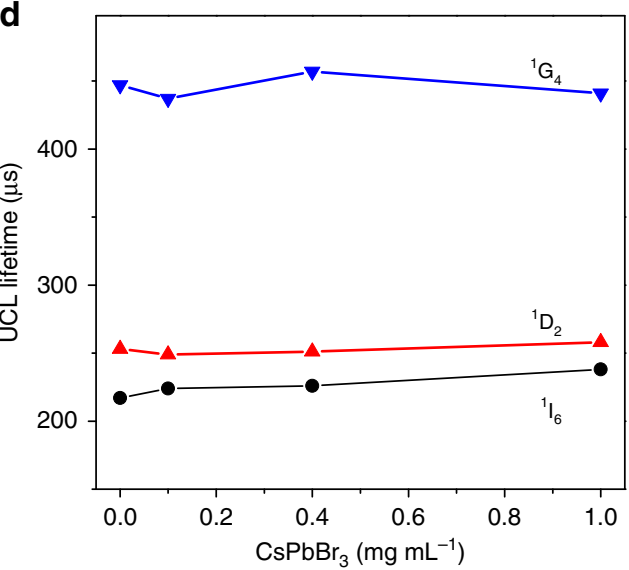

Fig. 4 Mechanistic investigation of the radiative energy transfer upconversion (RETU) process in nanoparticle (NP)-sensitized CsPbX ${ }_{3}$ perovskite quantum dots (PeQDs). a $\mathrm{CsPbBr}_{3}$ concentration-dependent upconversion luminescence (UCL) spectra for LiYbF $: 0.5 \% \mathrm{Tm}^{3+} @ \mathrm{LiYF}_{4}$ core/shell NP-sensitized $\mathrm{CsPbBr}_{3} \mathrm{PeQDs}$ with NPs concentration of $1 \mathrm{mg} \mathrm{mL}^{-1}$ under $980 \mathrm{~nm}$ excitation at a power density of $50 \mathrm{~W} \mathrm{~cm}^{-2}$. $\mathbf{b}$ Integrated intensities of the Tm $\mathrm{TH}^{3+}$ emissions at 347, 362, 450,483, and $648 \mathrm{~nm}$ and the $\mathrm{CsPbBr}_{3}$ emission at $520 \mathrm{~nm}$ vs. the $\mathrm{CsPbBr}_{3}$ concentration, as obtained from (a). c UCL decays from ${ }^{1} \mathrm{D}_{2}$ of $\mathrm{Tm}^{3+}$ by monitoring the $\mathrm{Tm}^{3+}$ emission at $362 \mathrm{~nm}$ in NP-sensitized $\mathrm{CsPbBr}_{3}$ PeQDs with varying $\mathrm{CsPbBr}_{3}$ concentrations under $980 \mathrm{~nm}$ excitation. d UCL lifetimes of ${ }^{1} \mathrm{I}_{6},{ }^{1} \mathrm{D}_{2}$, and ${ }^{1} \mathrm{G}_{4}$ of $\mathrm{Tm}^{3+}$ in NP-sensitized $\mathrm{CsPbBr}_{3} \mathrm{PeQDs}$ vs. the $\mathrm{CsPbBr}_{3}$ concentration

sensitize $\mathrm{CsPbBr}, \mathrm{CsPb}_{3}, \mathrm{CsPb}(\mathrm{Cl} / \mathrm{Br})_{3}$, or $\mathrm{CsPb}(\mathrm{Br} / \mathrm{I})_{3}$ PeQDs. These results show unambiguously that the upconverted exciton emissions in NP-sensitized PeQDs originate from the NPs-toPeQDs energy transfer. As shown in Fig. 3f, the energy transfer efficiency was calculated to increase from 65.5 to 96.6 and $99.9 \%$ as the halide composition changed from $\mathrm{Cl}^{-}$to $\mathrm{Br}^{-}$and $\mathrm{I}^{-}$(see Supplementary Note 2 and Supplementary Table 4 for details in definition and calculation). The lower efficiency of energy transfer from the NPs to $\mathrm{CsPbCl}_{3}$ can be attributed to the smaller absorbance of $\mathrm{CsPbCl}_{3}$ in UV than the other $\mathrm{CsPbX}_{3} \mathrm{PeQDs}$, as evidenced by their absorption spectra. The absolute upconversion QYs, upon excitation at $980 \mathrm{~nm}$ with a power density of $100 \mathrm{~W}$ $\mathrm{cm}^{-2}$, were determined to be $0.06 \pm 0.01 \%, 0.39 \pm 0.08 \%$, and $0.36 \pm 0.09 \%$ for exciton emissions in NP-sensitized $\mathrm{CsPbCl}_{3}$, $\mathrm{CsPbBr} 3$, and $\mathrm{CsPb}_{3} \mathrm{PeQDs}$, respectively (Supplementary Note 3 and Supplementary Table 5). The overall upconversion QYs of the NP-sensitized PeQDs are in the range of $0.33-0.45 \%( \pm 0.07$ $-0.13 \%)$, which are comparable to that $(0.49 \pm 0.13 \%)$ of pure $\mathrm{LiYbF}_{4}: 0.5 \% \mathrm{Tm}^{3+} @ \mathrm{LiYF}_{4}$ core/shell NPs (Supplementary Table 5). We can estimate the expected partial QYs of the excitons in NP-sensitized PeQDs as being the product of the upconversion QYs of pure NPs by the efficiency of energy transfer and by the PLQYs of PeQDs; in this way, we attained $0.08,0.38$, and $0.28 \%$ for the chloride, bromide, and iodide perovskites, respectively, in good agreement with the experimental values mentioned above. This confirms the high photon conversion efficiency in NP-sensitized PeQDs. Note that higher upconversion QYs for $\mathrm{CsPb}_{3} \mathrm{PeQDs}$ could be achieved by improving both upconversion QYs of the NPs and PLQYs of PeQDs.

Mechanistic investigation of the RETU process. In view of the core/shell structure of the NPs that imposed a distance of $5.5 \mathrm{~nm}$ between the energy donor and acceptor, we deduced that the energy transfer from the NPs to PeQDs is dominated by a radiative reabsorption process instead of a non-radiative FRET process, because the core/shell structure is unfavorable for distance-dependent FRET ${ }^{49,50}$. To gain more insights into the energy transfer process, we investigated PeQDs concentrationdependent steady-state and transient UCL in NP-sensitized PeQDs. As shown in Fig. 4a, under $980 \mathrm{~nm} \mathrm{CW}$ diode laser excitation, the $\mathrm{CsPBBr}_{3}$ emission at $520 \mathrm{~nm}$ increased gradually at the expense of the UV and blue emissions of $\mathrm{Tm}^{3+}$ with increasing $\mathrm{CsPbBr}_{3}$ concentration, as a result of energy transfer from the NPs to CsPbBr ${ }_{3}$ PeQDs. Coincidentally, the UV emission intensities of $\mathrm{Tm}^{3+}$ at $347 \mathrm{~nm}\left({ }^{1} \mathrm{I}_{6} \rightarrow{ }^{3} \mathrm{~F}_{4}\right)$ and $362 \mathrm{~nm}$ $\left({ }^{1} \mathrm{D}_{2} \rightarrow{ }^{3} \mathrm{H}_{6}\right)$ dropped much faster than the blue emissions at 450 $\mathrm{nm}\left({ }^{1} \mathrm{D}_{2} \rightarrow{ }^{3} \mathrm{~F}_{4}\right)$ and $483 \mathrm{~nm}\left({ }^{1} \mathrm{G}_{4} \rightarrow{ }^{3} \mathrm{H}_{6}\right)$ with increasing $\mathrm{Cs} \mathrm{PbBr}_{3}$ concentration, whereas the red emissions at $648 \mathrm{~nm}$ $\left({ }^{1} \mathrm{G}_{4} \rightarrow{ }^{3} \mathrm{~F}_{4}\right)$ remained nearly unchanged (Fig. $\left.4 \mathrm{~b}\right)$. This can be 

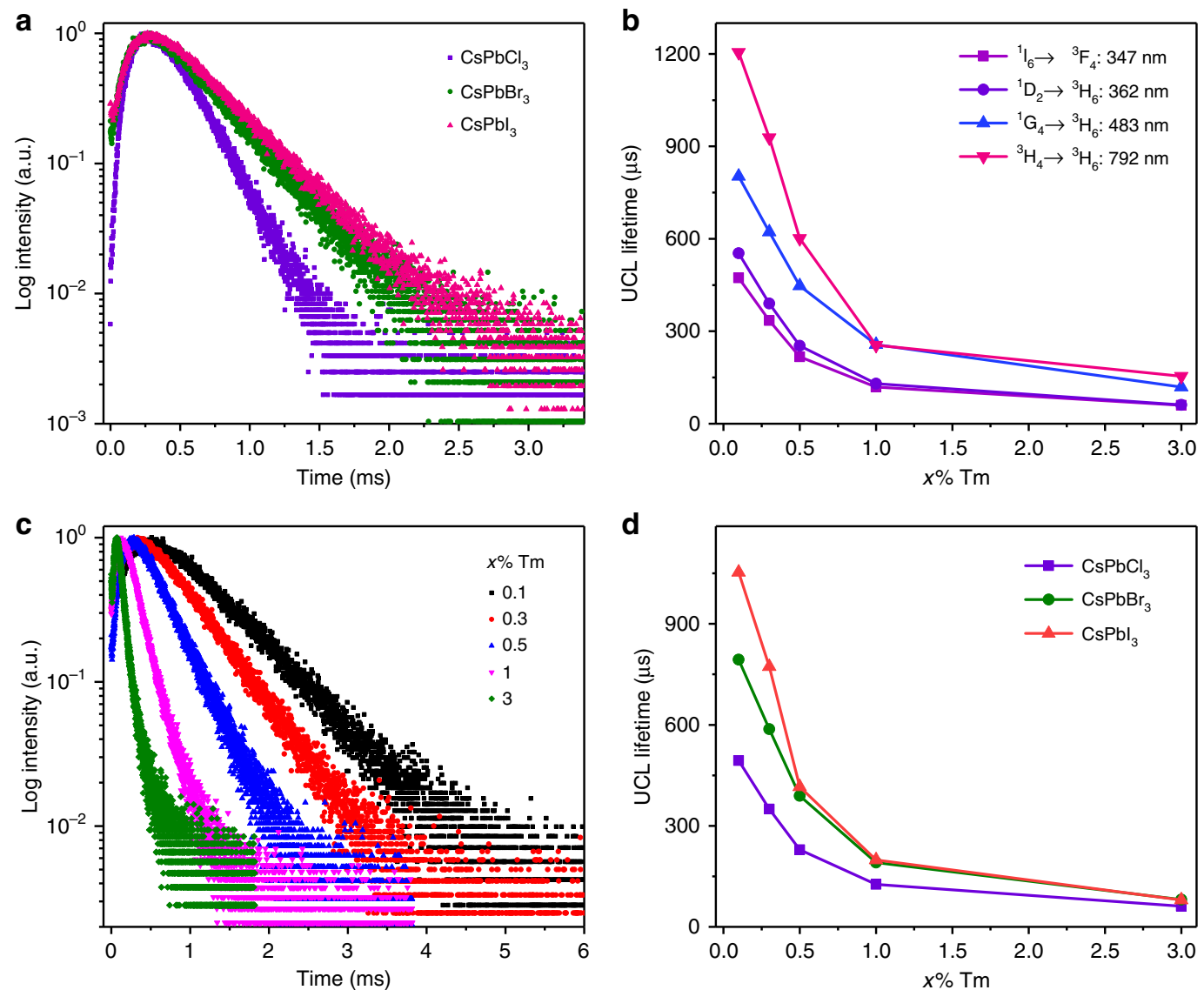

Fig. 5 Upconversion lifetime tuning in nanoparticle (NP)-sensitized $\mathrm{CsPbX}_{3}$ perovskite quantum dots (PeQDs). a Upconversion luminescence (UCL) decays from the upconverted excitons in NP-sensitized $\mathrm{CsPbCl}_{3}, \mathrm{CsPbBr}_{3}$, and $\mathrm{CsPbl}_{3} \mathrm{PeQDs}\left(0.5 \mathrm{~mol}^{2} \mathrm{Tm}^{3+}\right.$ ) by monitoring their emissions at 410 , 520 , and $700 \mathrm{~nm}$, respectively, under $980 \mathrm{~nm}$ excitation. b UCL lifetimes of ${ }^{1} \mathrm{l}_{6},{ }^{1} \mathrm{D}_{2},{ }^{1} \mathrm{G}_{4}$, and ${ }^{3} \mathrm{H}_{4}$ of $\mathrm{Tm}^{3+}$ in LiYbF $4: \mathrm{x} \mathrm{Tm}^{3+} @ \mathrm{LiYF}_{4}$ core/shell NPs as a function of the $\mathrm{Tm}^{3+}$ concentration. $\mathbf{c} \cup \mathrm{CL}$ decays from the upconverted excitons in NP-sensitized $\mathrm{CsPbBr}_{3} \mathrm{PeQDs}$ with varying $\mathrm{Tm}^{3+}$ concentration in the NPs. $\mathbf{d}$ UCL lifetimes of $\mathrm{CsPbCl}_{3}, \mathrm{CsPbBr}_{3}$, and $\mathrm{CsPb}_{3} \mathrm{PeQDs}$ as a function of the $\mathrm{Tm}^{3+}$ concentration

attributed to the much larger absorbance of $\mathrm{CsPbr}_{3}$ in UV than in blue and no absorption in red (Supplementary Fig. 9). One should keep in mind that the depopulation of ${ }^{1} \mathrm{D}_{2}$ or ${ }^{1} \mathrm{G}_{4}$ of $\mathrm{Tm}^{3+}$ through non-radiative FRET would lead to simultaneous quenching of all emissions from ${ }^{1} \mathrm{D}_{2}$ or ${ }^{1} \mathrm{G}_{4}{ }^{32}$. Thus, the observed distinct UCL evolutions between the emissions from identical ${ }^{1} \mathrm{D}_{2}$ or ${ }^{1} \mathrm{G}_{4}$ level reveal that the NPs-to-PeQDs energy transfer may be a radiative reabsorption process rather than a non-radiative FRET process. Such radiative energy transfer was further evidenced by the essentially unchanged UCL lifetimes of ${ }^{1} \mathrm{I}_{6},{ }^{1} \mathrm{D}_{2}$, and ${ }^{1} \mathrm{G}_{4}$ of $\mathrm{Tm}^{3+}$ in NP-sensitized $\mathrm{CsPbBr}_{3}$ PeQDs in comparison with those in NPs alone (Fig. 4c, d and Supplementary Fig. 10), because non-radiative FRET always results in a decrease in lifetime of energy donor by imposing additional relaxation channel on the donor ${ }^{51}$. The drastically distinct evolutions in PeQDs concentration-dependent UCL between the emissions from ${ }^{1} \mathrm{D}_{2}$ or ${ }^{1} \mathrm{G}_{4}$ level and the nearly unchanged UCL lifetimes of $\mathrm{Tm}^{3+}$ were also observed for PeQDs with different halide compositions (Supplementary Figs. 11-18), thus demonstrating that the upconverted exciton emission from the NP-sensitized $\mathrm{CsPbX}_{3}$ PeQDs is governed by a RETU process.

Upconversion lifetime tuning in NP-sensitized CsPbX $\mathrm{X}_{3} \mathrm{PeQDs}$. More importantly, we discovered that the UCL lifetimes of exciton emissions in NP-sensitized PeQDs were abnormally much longer (hundreds of $\mu$ s vs. ns) than their PL lifetimes in pure PeQDs. Figure 5a compares the UCL decays from the exciton emissions in NP-sensitized $\mathrm{CsPbCl}_{3}, \mathrm{CsPbBr}_{3}$, and $\mathrm{CsPbI}_{3}$ PeQDs, respectively, under $980 \mathrm{~nm}$ excitation. It was observed that all the decays displayed similar temporal profiles to that of $\mathrm{Tm}^{3+}$, namely, a rising edge in the initial stage followed by singleexponential decay, as a result of RETU process ${ }^{52}$. By singleexponential fitting to the decay curves, the UCL lifetimes of $\mathrm{CsPbCl}, \mathrm{CsPbBr}_{3}$, and $\mathrm{CsPbI}_{3}$ PeQDs were determined to be 229, 389 , and $416 \mu \mathrm{s}$, respectively, in marked contrast to their original PL lifetimes of 1.8, 21.1, and 81.1 ns under UV excitation (Supplementary Table 2). It is worth mentioning that the exciton lifetimes of PeQDs were intrinsically unchanged, but were apparently lengthened due to the slow population of the PeQDs excited state from the long-lived $\mathrm{Tm}^{3+}$ excited state during the radiative energy transfer from the NPs to PeQDs ${ }^{53,54}$. Therefore, through lifetime tuning of $\mathrm{Tm}^{3+}$ in the NPs, the apparent UCL lifetimes of PeQDs can be modulated. By controlling the $\mathrm{Tm}^{3+}$ concentration from $3 \mathrm{~mol} \%$ to $0.1 \mathrm{~mol} \%$ in the NPs, the lifetimes of ${ }^{1} \mathrm{I}_{6},{ }^{1} \mathrm{D}_{2},{ }^{1} \mathrm{G}_{4}$, and ${ }^{3} \mathrm{H}_{4}$ of $\mathrm{Tm}^{3+}$ increased from $60,61,119$, and $154 \mu$ s to $473,553,803$, and $1205 \mu$ s, respectively (Fig. 5b and Supplementary Fig. 19). Accordingly, the UCL lifetimes of $\mathrm{CsPbCl}{ }_{3}, \mathrm{CsPbBr}_{3}$, and $\mathrm{CsPbI}_{3} \mathrm{PeQDs}$ sensitized by the NPs were tuned from 61,81 , and $80 \mu$ s to 494,794 , and $1053 \mu$ s, respectively (Fig. 5c, d). Similar strategy was employed for manipulating the $\mathrm{UCL}$ lifetime of exciton in hybrid $\mathrm{CsPb}(\mathrm{Cl} / \mathrm{Br})_{3}$ and $\mathrm{CsPb}(\mathrm{Br} / \mathrm{I})_{3}$ PeQDs (Supplementary Figs. 20-27 and Supplementary Table 6), thus validating the general lifetime tunability in various PeQDs. The ability of ultralong lifetime tuning $(61 \mu \mathrm{s}-1.053 \mathrm{~ms})$ along 

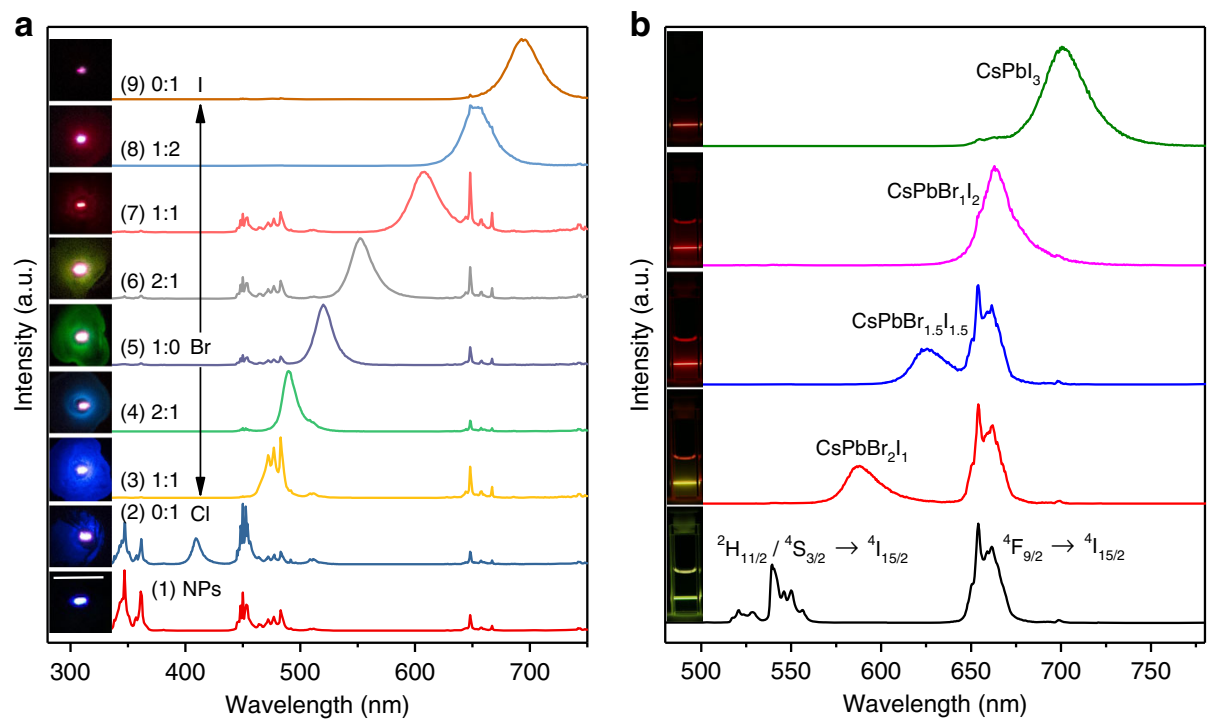

Fig. 6 Generality of the proposed radiative energy transfer upconversion (RETU) for sensitizing photon upconversion in $\mathrm{CsPbX}_{3}$ perovskite quantum dots (PeQDs). a Upconversion luminescence (UCL) spectra for $\mathrm{LiYbF}_{4}: 0.5 \% \mathrm{Tm}^{3+} @ \mathrm{LiYF}_{4}$ core/shell nanoparticles (NPs) and the NP-sensitized CsPbX $\mathrm{PeQDs}_{3}$ (NPs: $1 \mathrm{mg} \mathrm{mL}^{-1}$; PeQDs: $2 \mathrm{mg} \mathrm{mL}^{-1}$ ) with varying halide compositions by casting them onto glass substrate under $980 \mathrm{~nm} \mathrm{CW}$ diode laser excitation at a power density of $50 \mathrm{~W} \mathrm{~cm}^{-2}$. The insets show the corresponding $\mathrm{UCL}$ photographs for the NP-sensitized PeQDs, and the scale bar represents $1 \mathrm{~cm}$. $\mathbf{b} \mathrm{UCL}$ spectra for $\mathrm{NaYF}_{4}: 18 \% \mathrm{Yb}^{3+}, 2 \% \mathrm{Er}^{3+} \mathrm{NPs}$ (approximately $23 \mathrm{~nm}$ ) and the NP-sensitized CsPbX ${ }_{3}$ PeQDs (NPs: $1 \mathrm{mg} \mathrm{mL}^{-1} ; \mathrm{PeQDs}^{2} \mathrm{mg} \mathrm{mL}^{-1}$ ) with varying halide composition under $980 \mathrm{~nm} \mathrm{CW}$ diode laser excitation at a power density of $20 \mathrm{~W} \mathrm{~cm}^{-2}$. The insets show the corresponding $\mathrm{UCL}$ photographs for the colloidal cyclohexane solution of the NP-sensitized PeQDs

with wide color gamut modulation (140\% of the NTSC television color standard) via engineering the composition and concentration of PeQDs, offers us an unparalleled opportunity for constructing a huge library of discernable upconversion identities, which are of vital importance in optical encoding and multiplexing for applications in diverse areas such as complex data storage and multilevel anticounterfeiting ${ }^{55,56}$.

Generality of the proposed RETU. Furthermore, we demonstrated that the NP-sensitized PeQDs can be fabricated as films by casting them onto glass substrate, from which intense and tunable upconversion emissions were realized under $\mathrm{CW}$ diode laser excitation (Fig. 6a). The proposed RETU nanosystem can also be extended to the sensitization of different kinds of quantum dots by employing different lanthanide-doped NPs as energy donors. For example, through sensitization by $\mathrm{NaYF}_{4}: \mathrm{Yb}^{3+}, \mathrm{Er}^{3+} \mathrm{NPs}$, tunable upconverted exciton emissions were detected in $\mathrm{CsPbX}_{3}$ PeQDs (Fig. 6b). Similarly, upconverted exciton emissions from CdSe and InP@ZnS quantum dots were explicitly observed through radiative energy transfer from $\mathrm{LiYbF}_{4}: \mathrm{Tm}^{3+} @ \mathrm{LiYF}_{4}$ (Supplementary Figs. 28 and 29). These results show the generality of RETU for sensitizing photon upconversion in various quantum dots.

\section{Discussion}

In contrast to multiphoton absorption, the proposed RETU in $\mathrm{NP}$-sensitized $\mathrm{CsPbX}_{3} \mathrm{PeQDs}$ is made much more efficient through the use of physically existing intermediate energy levels of lanthanide ions and thus can be triggered by using a low-cost NIR laser diode. In comparison with non-radiative FRET, the RETU nanosystem breaks the distance restriction on energy transfer efficiency, and therefore is much more robust and convenient through simple mixing of lanthanide-doped NPs and PeQDs. Most importantly, as a merit of RETU, the PL lifetime of the exciton in PeQDs inheriting from the excited-state lifetime of lanthanides can be lengthened tremendously from the original nanosecond scale to milliseconds. Such a unique PL lifetime of the exciton is beneficial for electron and hole separation in photovoltaics by distributing the large number of excitons in a much longer time window, and is also highly desirable for timegated PL biosensing to eliminate the interference of short-lived background noise. Nevertheless, there remain many technical challenges to be overcome toward their practical applications, including the concerns about the stability and toxicity of PeQDs, as well as their upconversion efficiency. We envision that these concerns can be addressed by overall optimization involving the controlled synthesis and surface modification of the NPsensitized PeQDs. For example, we can encapsulate lanthanidedoped NPs and PeQDs into $\mathrm{SiO}_{2}$ or polystyrene NPs to improve the chemical stability, and employ dye-sensitized lanthanidedoped NPs as an antenna to broaden the excitation wavelengths and boost the upconversion efficiency. Finally, it is urgent to develop lead-free PeQDs with desirable physicochemical properties for optoelectronics and photovoltaics.

To conclude, the optical properties observed in lanthanidedoped NP-sensitized $\mathrm{CsPbX}_{3}$ PeQDs are striking because they have presented the first panorama of photon upconversion with high efficiency and multicolor and lifetime tunability in PeQDs under low power density excitation. These findings offer a general approach for fine tuning NIR-triggered photon upconversion in PeQDs, which may open up a new avenue for the exploration of PeQDs toward versatile applications, such as ultrasensitive bioassay, high-resolution bioimaging, and full-spectrum conversion in solar cells.

\section{Methods}

Chemicals and materials. $\mathrm{Pb}\left(\mathrm{CH}_{3} \mathrm{COO}\right)_{2} \cdot 3 \mathrm{H}_{2} \mathrm{O}(99.99 \%), \mathrm{CH}_{3} \mathrm{COOLi} \cdot 2 \mathrm{H}_{2} \mathrm{O}$ (99.99\%), $\mathrm{Cs}_{2} \mathrm{CO}_{3}$ (99.99\%), $\mathrm{HBr}(48 \%), \mathrm{HI}(55.0-58.0 \%)$ were purchased from Aladdin (Shanghai, China). Oleic acid (OA, 90\%), oleylamine (OAm, 90\%), 1octadecene (ODE, $90 \%)$, trioctylamine $(98 \%), \mathrm{Yb}\left(\mathrm{CH}_{3} \mathrm{COO}\right)_{3} \cdot 4 \mathrm{H}_{2} \mathrm{O}(99.999 \%), \mathrm{Tm}$ $\left(\mathrm{CH}_{3} \mathrm{COO}\right)_{3} \cdot 4 \mathrm{H}_{2} \mathrm{O}(99.99 \%)$, and $\mathrm{Y}\left(\mathrm{CH}_{3} \mathrm{COO}\right)_{3} \cdot 4 \mathrm{H}_{2} \mathrm{O}(99.99 \%)$ were purchased from Sigma-Aldrich (Shanghai, China). $\mathrm{NH}_{4} \mathrm{~F}, \mathrm{HCl}$, cyclohexane, acetone, and ethanol were of analytical grade and purchased from Sinopharm Chemical Reagent Co. (Shanghai, China). All the chemical reagents were used as received without further purification. 
Synthesis of $\mathbf{C s P b X}_{\mathbf{3}}$ PeQDs. Monodisperse $\mathrm{CsPbX}_{3}(\mathrm{X}=\mathrm{Cl}, \mathrm{Br}$, and I) PeQDs were synthesized through a modified hot-injection method by using $\mathrm{HX}$ as the halide source to precipitate the PeQDs ${ }^{45}$. As a result, the halide composition of the PeQDs can be precisely adjusted on demand by changing the ratio of HX during the synthesis. In a typical synthesis of $\mathrm{CsPbBr}_{3} \mathrm{PeQDs}, 0.5 \mathrm{mmol}$ of $\mathrm{Pb}$ $\left(\mathrm{CH}_{3} \mathrm{COO}\right)_{3} \cdot 3 \mathrm{H}_{2} \mathrm{O}$ and $0.1 \mathrm{mmol}$ of $\mathrm{Cs}_{2} \mathrm{CO}_{3}$ were mixed with $2 \mathrm{~mL}$ of $\mathrm{OA}, 2 \mathrm{~mL}$ of $\mathrm{OAm}$, and $6 \mathrm{~mL}$ of ODE in a $50 \mathrm{~mL}$ three-neck round-bottom flask. The resulting mixture was heated to $120^{\circ} \mathrm{C}$ under a $\mathrm{N}_{2}$ flow with constant stirring for $40 \mathrm{~min}$ to form a clear solution. The temperature was then raised up to $180^{\circ} \mathrm{C}$ and stabilized for $10 \mathrm{~min}$, and $1.5 \mathrm{mmol}$ of $\mathrm{HBr}$ was quickly injected. After $20 \mathrm{~s}$, the reaction mixture was cooled down to room temperature by ice-water bath. For the synthesis of $\mathrm{CsPbCl}_{3}$ and $\mathrm{CsPbI}_{3} \mathrm{PeQDs}, 1.5 \mathrm{mmol}$ of $\mathrm{HCl}$ and $\mathrm{HI}$ instead of $\mathrm{HBr}$ was injected, respectively, under otherwise identical conditions. For the synthesis of mixed-halide $\mathrm{CsPb}(\mathrm{Cl} / \mathrm{Br})_{3}$ and $\mathrm{CsPb}(\mathrm{Br} / \mathrm{I})_{3}$ PeQDs, $1.5 \mathrm{mmol}$ of total amount of mixed $\mathrm{HCl} / \mathrm{HBr}$ and $\mathrm{HBr} / \mathrm{HI}$ was injected, respectively. The final halide composition of the PeQDs was designated by the ratio of $\mathrm{HCl} / \mathrm{HBr} / \mathrm{HI}$ used in the synthesis.

Isolation and purification of $\mathbf{C s P b X} \mathbf{X}_{\mathbf{3}} \mathbf{P e Q D s}$. The crude solution was cooled down to room temperature with ice-water bath and the PeQDs were collected by centrifuging at 12,000 rpm for $5 \mathrm{~min}$. The precipitate was then dispersed in $1 \mathrm{~mL}$ of cyclohexane and centrifuged again at 12,000 rpm for $5 \mathrm{~min}$. After centrifugation, the supernatant was discarded and the PeQDs were redispersed in $30 \mathrm{~mL}$ of cyclohexane. Finally, $100 \mu \mathrm{L}$ of OAm was added and ultrasonicated for $1 \mathrm{~min}$ to stabilize the PeQDs for long-term storage.

Structural and optical characterization. Powder X-ray powder diffraction patterns of the samples were collected with an X-ray diffractometer (MiniFlex2, Rigaku) with $\mathrm{Cu}$ Kal radiation $(\lambda=0.154187 \mathrm{~nm})$. Both the low- and highresolution TEM measurements were performed by using a TECNAI G ${ }^{2}$ F20 TEM equipped with the energy dispersive X-ray spectrum. Optical absorption spectra of $\mathrm{CsPbX}_{3} \mathrm{PeQDs}$ were collected with a Perkin-Elmer Lambda365 UV/Vis spectrometer in transmission mode. PL excitation and emission spectra and PL decays were recorded on the FLS980 spectrometer (Edinburgh) equipped with both continuous xenon $(450 \mathrm{~W})$ and pulsed flash lamps. UCL emission spectra were acquired under $980 \mathrm{~nm}$ excitation with a CW diode laser ( $2 \mathrm{~W}$ ). UCL lifetimes were measured with a customized UV to mid-infrared steady-state and phosphorescence lifetime spectrometer (FSP920-C, Edinburgh) equipped with a digital oscilloscope (TDS3052B, Tektronix) and a tunable mid-band Optical Parametric Oscillator pulsed laser as the excitation source $(410-2400 \mathrm{~nm}, 10 \mathrm{~Hz}$, pulse width $\leq 5 \mathrm{~ns}$, Vibrant 355II, OPOTEK). PL photographs of the NPs and PeQDs were taken by using a Canon 70D digital camera without using any filter. The absolute PLQYs of $\mathrm{CsPbX}_{3} \mathrm{PeQDs}$ were measured by employing a standard barium sulfate coated integrating sphere (150 $\mathrm{mm}$ in diameter, Edinburgh) as the sample chamber that was mounted on the FLS920 spectrometer with the entry and output port of the sphere located in $90^{\circ}$ geometry from each other in the plane of the spectrometer. A standard tungsten lamp was used to correct the optical response of the instrument. All the spectral data were recorded at room temperature and corrected for the spectral response of both the spectrometer and the integrating sphere.

Data availability. The authors declare that all data supporting the findings of this study are available within the paper and its supplementary information files.

Received: 30 March 2018 Accepted: 19 July 2018

Published online: 27 August 2018

\section{References}

1. Swarnkar, A. et al. Colloidal $\mathrm{CsPbBr}_{3}$ perovskite nanocrystals: luminescence beyond traditional quantum dots. Angew. Chem. Int. Ed. 54, 15424-15428 (2015).

2. Bekenstein, Y., Koscher, B. A., Eaton, S. W., Yang, P. \& Alivisatos, A. P. Highly luminescent colloidal nanoplates of perovskite cesium lead halide and their oriented assemblies. J. Am. Chem. Soc. 137, 16008-16011 (2015).

3. De Roo, J. et al. Highly dynamic ligand binding and light absorption coefficient of cesium lead bromide perovskite nanocrystals. ACS Nano 10, 2071-2081 (2016).

4. Kovalenko, M. V., Protesescu, L. \& Bodnarchuk, M. I. Properties and potential optoelectronic applications of lead halide perovskite nanocrystals. Science 358, 745-750 (2017).

5. Song, J. et al. Quantum dot light-emitting diodes based on inorganic perovskite cesium lead halides $\left(\mathrm{CsPbX}_{3}\right)$. Adv. Mater. 27, 7162-7167 (2015).

6. Wang, Y. et al. All-inorganic colloidal perovskite quantum dots: a new class of lasing materials with favorable characteristics. Adv. Mater. 27, 7101-7108 (2015).
7. Saliba, M. et al. Incorporation of rubidium cations into perovskite solar cells improves photovoltaic performance. Science 354, 206-209 (2016).

8. Swarnkar, A. et al. Quantum dot-induced phase stabilization of $a-\mathrm{CsPI}_{3}$ perovskite for high-efficiency photovoltaics. Science 354, 92-95 (2016).

9. Huang, $\mathrm{H}$. et al. Water resistant $\mathrm{CsPbX}_{3}$ nanocrystals coated with polyhedral oligomeric silsesquioxane and their use as solid state luminophores in allperovskite white light-emitting devices. Chem. Sci. 7, 5699-5703 (2016).

10. He, X., Qiu, Y. \& Yang, S. Fully-inorganic trihalide perovskite nanocrystals: a new research frontier of optoelectronic materials. Adv. Mater. 29, 1700775 (2017).

11. Zhou, D. L. et al. Cerium and ytterbium codoped halide perovskite quantum dots: a novel and efficient downconverter for improving the performance of silicon solar cells. Adv. Mater. 29, 1704149 (2017).

12. Pan, G. et al. Doping lanthanide into perovskite nanocrystals: highly improved and expanded optical properties. Nano. Lett. 17, 8005-8011 (2017).

13. Deutsch, Z., Neeman, L. \& Oron, D. Luminescence upconversion in colloidal double quantum dots. Nat. Nanotechnol. 8, 649-653 (2013).

14. Pan, J. et al. Air-stable surface-passivated perovskite quantum dots for ultrarobust, single- and two-photon-induced amplified spontaneous emission. J. Phys. Chem. Lett. 6, 5027-5033 (2015).

15. Wang, Y. et al. Nonlinear absorption and low-threshold multiphoton pumped stimulated emission from all-inorganic perovskite nanocrystals. Nano. Lett. 16, 448-453 (2016).

16. Xu, Y. et al. Two-photon-pumped perovskite semiconductor nanocrystal lasers. J. Am. Chem. Soc. 138, 3761-3768 (2016).

17. Chen, W. et al. Giant five-photon absorption from multidimensional coreshell halide perovskite colloidal nanocrystals. Nat. Commun. 8, 15198 (2017).

18. Chen, J. S. et al. Size- and wavelength-dependent two-photon absorption cross-section of $\mathrm{CsPbBr}_{3}$ perovskite quantum dots. J. Phys. Chem. Lett. 8, 2316-2321 (2017)

19. Zou, W. Q., Visser, C., Maduro, J. A., Pshenichnikov, M. S. \& Hummelen, J. C. Broadband dye-sensitized upconversion of near-infrared light. Nat. Photon. 6 , 560-564 (2012).

20. Wang, J. et al. Photon energy upconversion through thermal radiation with the power efficiency reaching 16\%. Nat. Commun. 5, 5669 (2014).

21. Lee, J. et al. Universal process-inert encoding architecture for polymer microparticles. Nat. Mater. 13, 524-529 (2014).

22. Zhou, B., Shi, B. Y., Jin, D. Y. \& Liu, X. G. Controlling upconversion nanocrystals for emerging applications. Nat. Nanotechnol. 10, 924-936 (2015).

23. $\mathrm{Wu}, \mathrm{M}$. et al. Solid-state infrared-to-visible upconversion sensitized by colloidal nanocrystals. Nat. Photon. 10, 31-34 (2015).

24. Jalani, G. et al. Photocleavable hydrogel-coated upconverting nanoparticles: a multifunctional theranostic platform for nir imaging and on-demand macromolecular delivery. J. Am. Chem. Soc. 138, 1078-1083 (2016)

25. Kakavelakis, G., Petridis, K. \& Kymakis, E. Recent advances in plasmonic metal and rare-earth-element upconversion nanoparticle doped perovskite solar cells. J. Mater. Chem. A 5, 21604-21624 (2017).

26. Li, J. et al. Stable high-performance flexible photodetector based on upconversion nanoparticles/perovskite microarrays composite. ACS Appl. Mater. Interfaces 9, 19176-19183 (2017).

27. Auzel, F. Upconversion and anti-stokes processes with $\mathrm{f}$ and $\mathrm{d}$ ions in solids. Chem. Rev. 104, 139-173 (2004).

28. Venkataramanan, M., Fiorenzo, V., Rafik, N., Adolfo, S. \& A., C. J. Colloida $\mathrm{Tm}^{3+} / \mathrm{Yb}^{3+}$-doped $\mathrm{LiYF}_{4}$ nanocrystals: multiple luminescence spanning the UV to NIR regions via low-energy excitation. Adv. Mater. 21, 4025-4028 (2009).

29. Haase, M. \& Schafer, H. Upconverting nanoparticles. Angew. Chem. Int. Ed. 50, 5808-5829 (2011).

30. Zheng, W. et al. Lanthanide-doped upconversion nano-bioprobes: electronic structures, optical properties, and biodetection. Chem. Soc. Rev. 44, 1379-1415 (2015).

31. Bunzli, J.-C. G. \& Piguet, C. Taking advantage of luminescent lanthanide ions. Chem. Soc. Rev. 34, 1048-1077 (2005).

32. Wang, F. et al. Tuning upconversion through energy migration in core-shell nanoparticles. Nat. Mater. 10, 968-973 (2011).

33. Gargas, D. J. et al. Engineering bright sub-10-nm upconverting nanocrystals for single-molecule imaging. Nat. Nanotechnol. 9, 300-305 (2014).

34. Quintanilla, M., Ren, F., Ma, D. \& Vetrone, F. Light management in upconverting nanoparticles: ultrasmall core/shell architectures to tune the emission color. ACS Photonics 1, 662-669 (2014).

35. Han, S. Y. et al. Multicolour synthesis in lanthanide-doped nanocrystals through cation exchange in water. Nat. Commun. 7, 13059 (2016).

36. Yan, C. L., Zhao, H. G., Perepichka, D. F. \& Rosei, F. Lanthanide ion doped upconverting nanoparticles: synthesis, structure and properties. Small 12, 3888-3907 (2016) 
37. Gnach, A. \& Bednarkiewicz, A. Lanthanide-doped up-converting nanoparticles: merits and challenges. Nano Today 7, 532-563 (2012).

38. Yan, C., Dadvand, A., Rosei, F. \& Perepichka, D. F. Near-IR photoresponse in new up-converting $\mathrm{CdSe} / \mathrm{NaYF}_{4}: \mathrm{Yb}$,Er nanoheterostructures. J. Am. Chem. Soc. 132, 8868-8869 (2010).

39. Bednarkiewicz, A., Nyk, M., Samoc, M. \& Strek, W. Up-conversion FRET from $\mathrm{Er}^{3+} / \mathrm{Yb}^{3+}: \mathrm{NaYF}_{4}$ nanophosphor to CdSe quantum dots. J. Phys. Chem. C. 114, 17535-17541 (2010).

40. Marin, R. et al. Upconverting nanoparticle to quantum dot Förster resonance energy transfer: increasing the efficiency through donor design. ACS Photonics 5, 2261-2270 (2018).

41. Akkerman, Q. A. et al. Tuning the optical properties of cesium lead halide perovskite nanocrystals by anion exchange reactions. J. Am. Chem. Soc. 137, 10276-10281 (2015).

42. Ravi, V. K., Markad, G. B. \& Nag, A. Band edge energies and excitonic transition probabilities of colloidal $\mathrm{CsPbX}_{3}(\mathrm{X}=\mathrm{Cl}, \mathrm{Br}, \mathrm{I})$ perovskite nanocrystals. ACS Energy Lett. 1, 665-671 (2016).

43. Zou, Q. et al. Cooperative and non-cooperative sensitization upconversion in lanthanide-doped $\mathrm{LiYbF}_{4}$ nanoparticles. Nanoscale 9, 6521-6528 (2017).

44. Pollnau, M., Gamelin, D. R., Luthi, S. R., Güdel, H. U. \& Hehlen, M. P. Power dependence of upconversion luminescence in lanthanide and transitionmetal-ion systems. Phys. Rev. B 61, 3337-3346 (2000).

45. Protesescu, L. et al. Nanocrystals of cesium lead halide perovskites $\left(\mathrm{CsPbX}_{3}\right.$, $\mathrm{X}=\mathrm{Cl}, \mathrm{Br}$, and I): novel optoelectronic materials showing bright emission with wide color gamut. Nano Lett. 15, 3692-3696 (2015).

46. Gorris, H. H. \& Wolfbeis, O. S. Photon-upconverting nanoparticles for optical encoding and multiplexing of cells, biomolecules, and microspheres. Angew. Chem. Int. Ed. 52, 3584-3600 (2013).

47. Dai, X. et al. Solution-processed, high-performance light-emitting diodes based on quantum dots. Nature 515, 96-99 (2014).

48. Deng, R. R. et al. Temporal full-colour tuning through non-steady-state upconversion. Nat. Nanotechnol. 10, 237-242 (2015).

49. Zhang, J., Fu, Y., Chowdhury, M. H. \& Lakowicz, J. R. Enhanced Förster resonance energy transfer on single metal particle. 2. Dependence on donoracceptor separation distance, particle size, and distance from metal surface. J. Phys. Chem. C. 111, 11784-11792 (2007).

50. Su, Q. Q., Feng, W., Yang, D. P. \& Li, F. Y. Resonance energy transfer in upconversion nanoplatforms for selective biodetection. Acc. Chem. Res. 50, 32-40 (2017)

51. Tu, D. T. et al. Time-resolved FRET biosensor based on amine-functionalized lanthanide-doped $\mathrm{NaYF}_{4}$ nanocrystals. Angew. Chem. Int. Ed. 50, 6306-6310 (2011).

52. Luo, W. Q. et al. $\mathrm{Er}^{3+}$-doped anatase $\mathrm{TiO}_{2}$ nanocrystals: crystal-field levels, excited-state dynamics, upconversion, and defect luminescence. Small 7, 3046-3056 (2011).

53. Cardoso Dos Santos, M. \& Hildebrandt, N. Recent developments in lanthanide-to-quantum dot FRET using time-gated fluorescence detection and photon upconversion. TrAC-Trend Anal. Chem. 84, 60-71 (2016).

54. Díaz, S. A. et al. Bridging lanthanide to quantum dot energy transfer with a short-lifetime organic dye. J. Phys. Chem. Lett. 8, 2182-2188 (2017).

55. Zhang, F. et al. Fluorescence upconversion microbarcodes for multiplexed biological detection: nucleic acid encoding. Adv. Mater. 23, 3775-3779 (2011).
56. Lu, Y. Q. et al. Tunable lifetime multiplexing using luminescent nanocrystals. Nat. Photon. 8, 33-37 (2014)

\section{Acknowledgements}

This work is supported by the 973 program of MOST (no. 2014CB845605), the Strategic Priority Research Program of the Chinese Academy of Sciences (XDB20000000), the NSFC (nos. 21325104, 21771185, 11774345, U1305244, 21501180, and 11704380), the CAS/SAFEA International Partnership Program for Creative Research Teams, the CAS Youth Innovation Promotion Association (no. 2016277), the Chunmiao Project of Haixi Institutes of the CAS (no. CMZX-2016-002), and Natural Science Foundation of Fujian Province (no. 2017I0018).

\section{Author contributions}

W.Z. and X.C. conceived the projects, wrote the paper, and were primarily responsible for the experiments. P.H., D.T., J.X., and J.-C.G.B. provided input into the design of the experiments. Z.G. and Q.Z. synthesized and characterized the NPs and PeQDs. R.L. and W.Y. carried out the QY measurement and calculation. All authors contributed to the analysis of the manuscript.

\section{Additional information}

Supplementary Information accompanies this paper at https://doi.org/10.1038/s41467 018-05947-2.

Competing interests: The authors declare no competing interests.

Reprints and permission information is available online at http://npg.nature.com/ reprintsandpermissions/

Publisher's note: Springer Nature remains neutral with regard to jurisdictional claims in published maps and institutional affiliations.

cc (i) Open Access This article is licensed under a Creative Commons Attribution 4.0 International License, which permits use, sharing, adaptation, distribution and reproduction in any medium or format, as long as you give appropriate credit to the original author(s) and the source, provide a link to the Creative Commons license, and indicate if changes were made. The images or other third party material in this article are included in the article's Creative Commons license, unless indicated otherwise in a credit line to the material. If material is not included in the article's Creative Commons license and your intended use is not permitted by statutory regulation or exceeds the permitted use, you will need to obtain permission directly from the copyright holder. To view a copy of this license, visit http://creativecommons.org/ licenses/by/4.0/.

(C) The Author(s) 2018 